5. Tan J, Gao W, Yang W, Zeng X, Wang L, Cui X. Isoform-specific functions of c-Jun N-terminal kinase 1 and 2 in lung ischemia-reperfusion injury through the c-Jun/activator protein-1 pathway. J Thorac Cardiovasc Surg. 2021;162: e143-56.

\section{Commentary: A tale of two} isoforms in lung ischemia reperfusion injury: One is bad, two is good

\author{
Jonathan D'Cunha, $\mathrm{MD}, \mathrm{PhD}$
}

In the spring of 1987, I was attending a lecture for Biocore Semester 2 at the University of Wisconsin introducing the concept of differential regulation of second messenger systems within the cell. Yes, I know I am dating myself, but this historical context is important for introducing the point of the decades of research into these pathways. At that time, cAMP was all the rage, and we were taught that understanding the signaling mechanisms within a cell and the downstream consequences of various stimuli would lead to better understanding of biological effects at higher levels. The molecular events leading to these cellular events humble clinicians, especially cardiothoracic surgeons, when treating a patient with hypoxia and systemic inflammation.

For patients with advanced lung disease, lung transplantation (LT) is a key therapeutic option. LT can indeed extend and improve the quality of life, but one of the major obstacles for successful long-term outcome is primary graft dysfunction (PGD) around the time of transplantation. PGD affects approximately $10 \%$ to $15 \%$ of all LT recipients and is associated with increased risks of chronic rejection and mortality. ${ }^{1}$ Characterized clinically and radiographically by

From the Department of Cardiothoracic Surgery, Mayo Clinic Arizona, Phoenix, Ariz.

Disclosures: The author reported no conflicts of interest.

The Journal policy requires editors and reviewers to disclose conflicts of interest and to decline handling or reviewing manuscripts for which they may have a conflict of interest. The editors and reviewers of this article have no conflicts of interest.

Received for publication April 26, 2020; revisions received April 26, 2020; accepted for publication April 29, 2020; available ahead of print May 13, 2020.

Address for reprints: Jonathan D'Cunha, MD, PhD, Department of Cardiothoracic Surgery, Mayo Clinic Arizona, 5777 E Mayo Blvd, Phoenix, AZ 85054 (E-mail: DCunha.Jonathan@mayo.edu).

J Thorac Cardiovasc Surg 2021;162:e158-9

$0022-5223 / \$ 36.00$

Copyright (c) 2020 by The American Association for Thoracic Surgery

https://doi.org/10.1016/j.jtcvs.2020.04.145

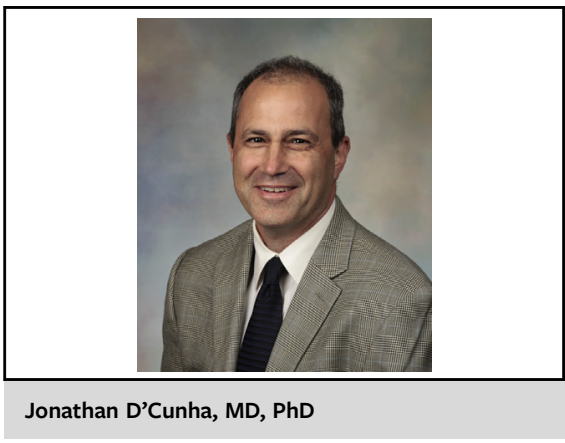

CENTRAL MESSAGE

The isoforms of c-Jun N-terminal

kinase 1 and 2 have different

functional impacts on ischemia/

reperfusion injury in a lung

transplantation model.

hypoxemia and pulmonary infiltrates (ie, edema) with diffuse alveolar damage, PGD is a severe form of ischemia/reperfusion (I/R) lung injury with inflammation occurring within the first 72 hours after lung transplantation.

$\mathrm{I} / \mathrm{R}$ injury (IRI) is multifactorial, complex, and highly integrated, leading to perturbation induced by ischemia and severe damage to cellular structures during reperfusion. These events lead to cell death and severe injury to transplanted organs. ${ }^{1}$ The incidence of I/R injury is much higher, such that it would be reasonable to postulate that it exists along a spectrum of severity. Despite considerable progress during the past decade, the molecular mechanisms responsible for IRI/PGD remain poorly understood. Consequently, identifying new molecular targets and preventive measures for IRI is critical for improved outcomes.

In this issue of the Journal, Tan and colleagues ${ }^{2}$ investigate the role of the c-Jun $\mathrm{N}$ terminal kinase (JNK) pathway and its role in IRI injury in a rat model of LT. JNK is a member of the mitogen-activated protein kinase (MAPK) family of signaling pathways within the cell that respond to stressful stimuli. Activated JNK exerts its influence through the AP1 family of transcription factors to regulate numerous cellular events, including proliferation, differentiation, inflammation, and survival. JNK is a 
stress-related pathway that is activated by proinflammatory cytokines and growth factors in response to such stimuli as hypoxia with reoxygenation. ${ }^{3}$ These pathways have been investigated for decades, and much is known about them.

I read this work with great interest because of the intriguing series of experiments and the clarity of the molecular findings. ${ }^{3}$ Using their previous published experience, the authors dissected their in vitro model of IRI to define the isoform response of JNKs with small-interfering RNAs. They found that inhibition of JNK1 was protective of the cells from apoptosis. ${ }^{3,4}$ The opposite was true in experiments with inhibition of JNK2. This work was carried into a rat model of LT with intratracheal injection of JNK1 silencing RNAs. In vivo, the authors observed that JNK1 silencing inhibited inflammation and apoptosis, thereby alleviating graft injury. These findings were supported not only functionally, but also histologically. Their work is quite convincing for a damaging role of JNK1 in IRI. Importantly, JNK2 appears to be protective. These are exciting experiments taken from the cell culture dish to an animal model. Assessing the clinical impact is an area for future studies.

As it relates to IRI and lung transplantation, this work has the potential to be extended into a growing body of literature that seeks to dissect the molecular events and intervene for this complex problem. The application of this molecular understanding will bring hope for potential therapeutics in the donor operating room, ex vivo perfusion, and the recipient operating room, with the overall goal of potentially improving the outcomes for this serious, unresolved clinical issue.

\section{References}

1. de Perrot M, Liu M, Waddell TK, Keshavjee S. Ischemia-reperfusion-induced lung injury. Am J Respir Crit Care Med. 2003;167:490-511.

2. Tan J, Gao W, Yang W, Zeng X, Wang L, Cui X. Isoform-specific functions of c-Jun N-terminal kinase 1 and 2 in lung ischemia-reperfusion injury through the c-Jun/activator protein-1 pathway. J Thorac Cardiovasc Surg. 2021;162:e143-56.

3. Eltzschig HK, Eckle T. Ischemia and reperfusion from mechanism to translation. Nat Med. 2011;17:1391-401.

4. Tan J, Liu D, Lv X, Wang L, Zhao C, Che Y, et al. MAPK mediates inflammatory response and cell death in rat pulmonary microvascular endothelial cells in an ischemia reperfusion model of lung transplantation. J Heart Lung Transplant. 2013;32:823-31.

\section{See Article page e143.}

\section{Commentary: Constant dripping wears a stone away}

\author{
Naoki Ozeki, MD, PhD, and \\ Toyofumi Fengshi Chen-Yoshikawa, MD, PhD
}

Lung transplantation is one of the last treatment options for end-stage respiratory insufficiency. Although postoperative outcomes of lung transplantation have improved over time, pulmonary ischemia-reperfusion injury (IRI) remains a serious concern. ${ }^{1,2}$ Primary graft dysfunction (PGD), a major cause of death in the early period after lung transplantation, is often related to pulmonary IRI. Prevention of PGD is

\footnotetext{
From the Department of Thoracic Surgery, Nagoya University Graduate School of Medicine, Nagoya, Japan.

Disclosures: The authors reported no conflicts of interest.

The Journal policy requires editors and reviewers to disclose conflicts of interest and to decline handling or reviewing manuscripts for which they may have a conflict of interest. The editors and reviewers of this article have no conflicts of interest.

Received for publication April 27, 2020; revisions received April 27, 2020; accepted for publication April 27, 2020; available ahead of print May 11, 2020.

Address for reprints: Toyofumi Fengshi Chen-Yoshikawa, MD, PhD, Department of Thoracic Surgery, Graduate School of Medicine, Nagoya University, 65 Tsurumaicho, Showa-ku, Nagoya, Japan (E-mail: tyoshikawa@med.nagoya-u.ac.jp).

J Thorac Cardiovasc Surg 2021;162:e159-60

$0022-5223 / \$ 36.00$

Copyright (c) 2020 by The American Association for Thoracic Surgery

https://doi.org/10.1016/j.jtcvs.2020.04.136
}

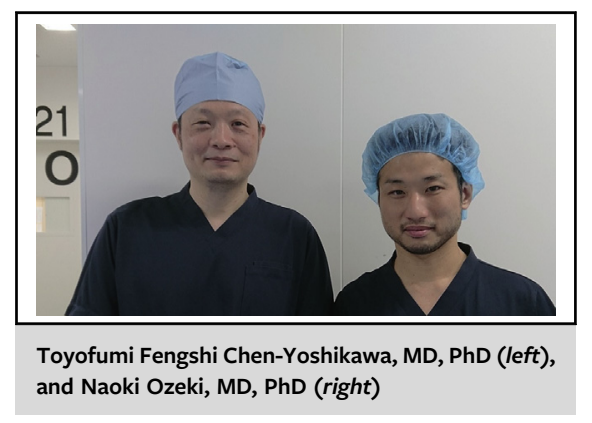

CENTRAL MESSAGE

A mitogen-activated protein

kinase, c-Jun N-terminal kinase

(JNK), plays a key role in lung

ischemia-reperfusion injury.

The opposite roles of JNK

isoforms are investigated for

clinical application.

imperative to achieve long-term graft survival; therefore, minimizing pulmonary IRI is one of the most important tasks related to lung transplantation. In this issue of the Journal, Tan and colleagues ${ }^{3}$ take on this challenging task. 